\title{
LESSONS FROM STANDARD CHARM DECAYS
}

\author{
J. M. DE MIRANDA \\ Centro Brasileiro de Pesquisas Fisicas, Rua Xavier Sigaud 150, Rio de Janeiro RJ 22290-180, Brazil \\ E-mail: jussara@cbpf.br
}

Recent charm results are reviwed with special attention to spectroscopy and hadronic decays. Two new states decaying to $D_{s}^{+} \pi^{0}$ and $D_{s}^{*+} \pi^{0}$ were recently discovered by BaBar and CLEO. In the baryon sector, the first observation of a doubly charmed baryon was announced by SELEX. Several amplitude analysis on charm hadronic decays are also discussed.

\section{Introduction}

Charm physics does not hold the frontier physics place that it once did, nevertheless there is still plenty of room for surprises and much to be understood. There is a consensus that charm is not a "heavy quark" (HQ) in the sense that the "heavy quark" QCD methodology would always work. Rather, charm seems to behave sometimes as a true heavy quark and sometimes not. That is why a large number of theoretical concepts and tools used in high energy physics are relevant for treating charm physics. Due to the richness of the decay pattern and the large clean samples now available charm physics is a very active/attractive research field. B-factories and collider experiments produced some impressive charm data, revealing large potential for high precision and rare processes in charm physics.

Very recently S. Bianco, F. L. Fabbri, D. Benson and I. Bigi wrote a quite complete and self-contained charm physics review. ${ }^{1}$ Both theoretical and experimental state-of-the-art aspects of the charm physics are carefully addressed. Here, due to the space restriction, I was forced to impose some stringent selection criteria.

I just mention two results that could not be better discussed. First, I summarize lifetime measurements of all charm hadrons in Fig. 1. The lifetimes are the most inclusive possible decay quantity, they carry information on the underlying dynamics of the weak decays. Although the lifetime hierarchy has been established theoretically by the use of $1 / m_{Q}$ expansions plus QCD corrections techniques, a more fundamental quantitative explanation is still necessary. The ability to make high precision lifetime measurements is essential for the search for mixing and possibly new physics in the charm sector. Traditionally the lifetime measurements have been made

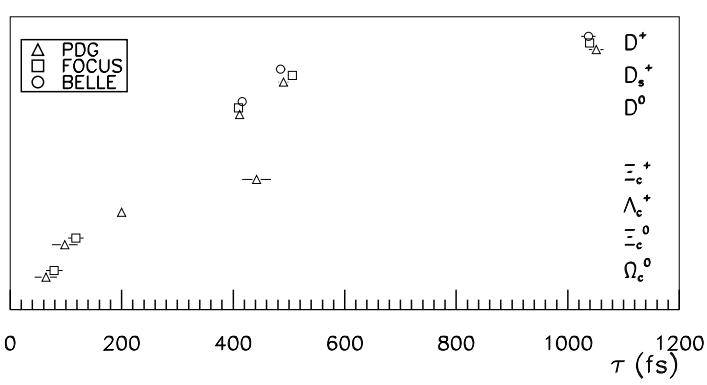

Figure 1. Charm lifetime measurements. Explicitly plotted are FOCUS and Belle measurements which are not yet included in the PDG averages.

by fixed target experiments, favored by the Lorentz boost that enlarges the production-decay displacements, but lately collider experiments have become competitive as is clear from Fig. 1.

The second result that I would like to at least mention is the observation of an unexpected interference phenomena in the semileptonic decay $D^{+} \rightarrow$ $K^{-} \pi^{+} \mu^{+} \nu$ by the FOCUS experiment. ${ }^{2}$ For the past 20 years this decay was believed to occur $100 \%$ through the intermediate $D^{+} \rightarrow \bar{K}^{* 0}(892) \mu^{+} \nu$. During the form factors analysis, FOCUS observed a large discrepancy between data and Monte Carlo. The noticed mismatch was significant only for events in the lower $K \pi$ mass region $\left(m_{K \pi}<0.9 \mathrm{GeV}\right)$. The simplest explanation for the effect would be the inclusion of a constant scalar amplitude that interferes with the dominant $\bar{K}^{* 0}$. Such an amplitude is parameterized as $A e^{i \delta}, A$ and $\delta$ being constants with best values measured to be 0.36 and $45^{\circ}$ respectively. This solution is plotted in Fig. 2.

The topics I selected to discuss more extensively are: spectroscopy (Sec. 1) and hadronic decays (Sec. 2). 


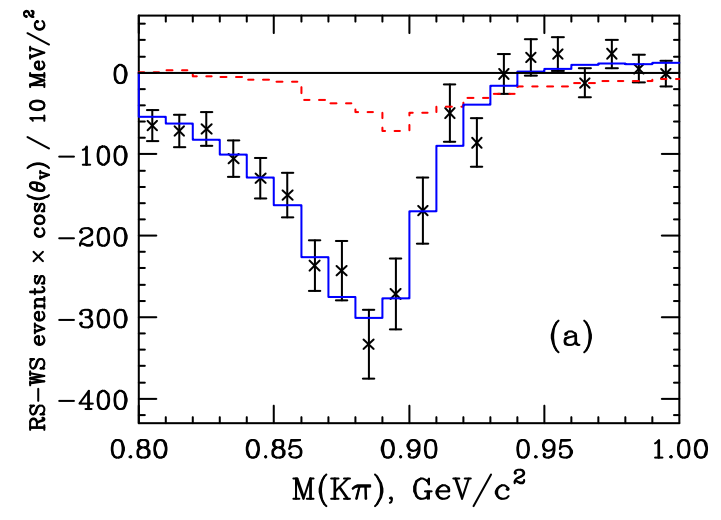

Figure 2. FOCUS $D^{+} \rightarrow K^{-} \pi^{+} \mu^{+} \nu$ asymmetry vs. $K \pi$ invariant mass. The dashed line represents the Monte Carlo simulation with no interfering S-wave. The solid line is the Monte Carlo with constant S-wave amplitude.

Models based on Heavy Quark Symmetry (HQS) were able to predict the first excited states for $[c \bar{u}]$ and $[c \bar{d}]$ and also some of the $[c \bar{s}]$ mesons. Last April, $\mathrm{BaBar}^{3}{ }^{3} \mathrm{CLEO}^{4}$ and later Belle ${ }^{5}$ observed two states decaying predominantly to $D_{s}^{+} \pi^{0}$ and $D_{s}^{*+} \pi^{0}$. Several complementary measurements were performed that favored the interpretation of these being the $\mathrm{L}=1 J^{P}=0^{+}, 1^{+}$states. This result was a surprise due to the much lower than expected mass observed.

Much more numerous, complex and less studied than the mesons, the baryon spectroscopy sector has also gained some visibility lately with a series of double charm meson states claimed by SELEX. ${ }^{6,7}$ Even though some of the proposed states are questionable due to the low statistics, they are worth noticing.

The last selected topic are the hadronic decays, with emphasis on full amplitude analysis. The investigation offers insight into a large number of issues: QCD, on heavy to low quark transitions, lifetime differences, final-state interactions, relative strength on the various decay diagrams and, more recently, it has proved to be a very interesting source of information on light meson spectroscopy. There is no overall interpretation as to why the hadronic charm decay pattern is what it is.

Many results shown here are preliminary results presented in conferences.

\section{Spectroscopy}

\subsection{Mesons}

Before last April, when the two states $D_{s J}^{*}(2317)$ $D_{s J}^{*}(2463)$ were observed by BaBar ${ }^{3}$ and CLEO,${ }^{4}$ the charm meson spectroscopy was reasonably well understood. The two above signals were first observed in $e^{+} e^{-}$annihilations in the decays to $D_{s}^{+} \pi^{0}$ and $D_{s}^{*+} \pi^{0}$ respectively. Later Belle ${ }^{5}$ also confirmed the results and made the first observation of the channel $D_{s J}^{*}(2463) \rightarrow D_{s} \gamma$ and several exclusive $B \rightarrow D D_{s J}^{*}$.

The charm meson ground states fit in the $\mathrm{SU}(4)_{\text {flavor }}$ multiplet classification scheme which is not useful for predicting the excited states due to large symmetry breaking $\left(m_{c} \ll m_{s}\right)$. The use of the Heavy Quark Symmetry (HQS) along with QCD based potential models provides a much more useful classification/prediction scheme. In the HQS the charmed meson $[Q \bar{q}]$ is viewed like the hydrogen atom. In the limit $m_{c} \rightarrow \infty$ the spin of the heavy quark $\left(S_{Q}\right)$ decouples from the light quark degrees of freedom, which means that $j_{q} \equiv S_{q}+L$ and $S_{Q}$ are separately conserved. Figure 3 summarizes the $[c \bar{d}]$ and $[c \bar{u}]$ spectroscopy lines. The four $L=1, n=1$ particles were observed experimentally. For parity and angular momentum conservations, $j_{q}=1 / 2$ states are forced to decay via $\mathrm{S}$-wave transitions and for that reason they have large widths while the $j_{q}=3 / 2$, that decay via $\mathrm{D}$-wave, are narrow states and much easier to observe. In Fig. 3 many experimental results are plotted, of those I emphasize the measurements made recently by Belle. ${ }^{8}$ Belle observed the four excited states by doing the full amplitude analysis of the decays $B^{-} \rightarrow D^{(*)+} \pi^{-} \pi^{-}$, which is the same procedure advocated in the next session to study light hadron spectroscopy with Dalitz plot analyses of charmed mesons.

Theory predicts also the existence of two radial excitations $(L=0, n=2)$, only one of which was seen by Delphi $\left(D^{* \prime}(2637)\right)^{9}$ but not confirmed by a number of other experiments. ${ }^{10}$

The use of the heavy quark symmetry for the $[c \bar{s}]$ excited states means a picture very similar to Fig. 3, only scaled up about $80 \mathrm{MeV}$. The narrow states have been observed in $D K$ decay modes with mass and width close to the theoretical prediction, see Table 1.

This scenario led to the belief that the states $D_{s 0}^{*}$ and $D_{s 1}^{*}\left(j_{q}=1 / 2\right)$ expected at masses $\sim 2480$ and $\sim 2570 \mathrm{MeV}$ respectively, would decay through $\mathrm{S}$-wave transitions in the isospin conserving modes $D K$, and consequently have large widths. Instead, $\mathrm{BaBar}^{3}$ announced the observation of a very narrow state, $D_{s J}^{*}(2317)$ at about $2.32 \mathrm{GeV}$ in the in- 


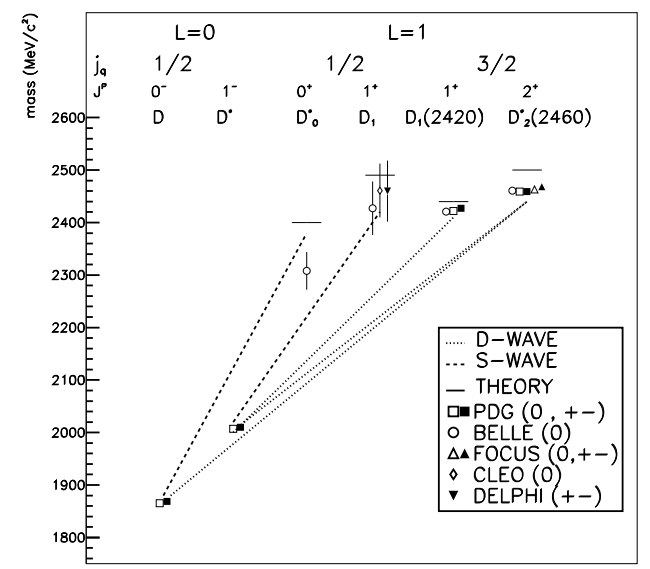

Figure 3. Spectroscopy scheme for $D^{0, \pm}$ mesons.

Table 1. Excited $[c \bar{s}]$ meson $D_{S J}^{*}\left(j_{q}\right)$. FOCUS preliminary results. ${ }^{11}$

\begin{tabular}{|c|c|c|c|}
\hline & Theory & PDG & FOCUS \\
\hline$D_{s 1}^{*}(2536)(3 / 2)$ & & & \\
$m(\mathrm{MeV})$ & $\sim 2530$ & $2573.3 \pm 6$ & $2535.1 \pm 0.3$ \\
$\Gamma(\mathrm{MeV})$ & $<1$ & $<2.390 \% \mathrm{CL}$ & $1.6 \pm 1$ \\
\hline$D_{s 2}^{*}(2573)(3 / 2)$ & & & \\
$m(\mathrm{MeV})$ & $\sim 2590$ & $2572.4 \pm 1.5$ & $2567.3 \pm 1.4$ \\
$\Gamma(\mathrm{MeV})$ & $10-20$ & $15 \pm 5$ & $28 \pm 5$ \\
\hline
\end{tabular}

clusive invariant mass of $D_{s}^{+} \pi^{0}$. They also noticed an excess of events at the $\sim 2.46 \mathrm{GeV}$ region of the $D^{*}(2112)^{+} \pi^{0}$ mass spectrum. This result triggered theoretical ${ }^{12}$ and experimental activities to understand, confirm and establish the properties of the new states. CLEO ${ }^{4}$ confirmed the $D_{s J}^{*}(2317)$ and claimed the existence of a new state $D_{s J}^{*}(2463)$ decaying to $D^{*}(2112)^{+} \pi^{0}$. Belle confirmed these prior results and observed the radiative decay mode $D_{s J}^{*}(2463) \rightarrow D_{s}^{+} \gamma$ and a series of exclusive $B \rightarrow$ $D D_{s J}^{*}$, from which they obtained some information on the spin of the new particles. ${ }^{5}$

Using Monte Carlo the three experiments excluded the possibility of any known particle to produce the observed signals. On the other hand the two new states are kinetically very similar, both mass differences $\Delta M_{D_{s J}^{*}(2317)} \equiv M_{D_{s} \pi^{0}}-M_{D_{s}}$ and $\Delta M_{D_{s J}^{*}(2463)} \equiv M_{D_{s}^{*} \pi^{0}}-M_{D_{s}^{*}}$ are of the order of $350 \mathrm{MeV}$. It is possible that $D_{s J}^{*}(2317)$ feeds-up to $D_{s J}^{*}(2463)$ by the addition of a random photon consistent with $D^{*}(2112)^{+} \rightarrow D_{s} \gamma$ or that the
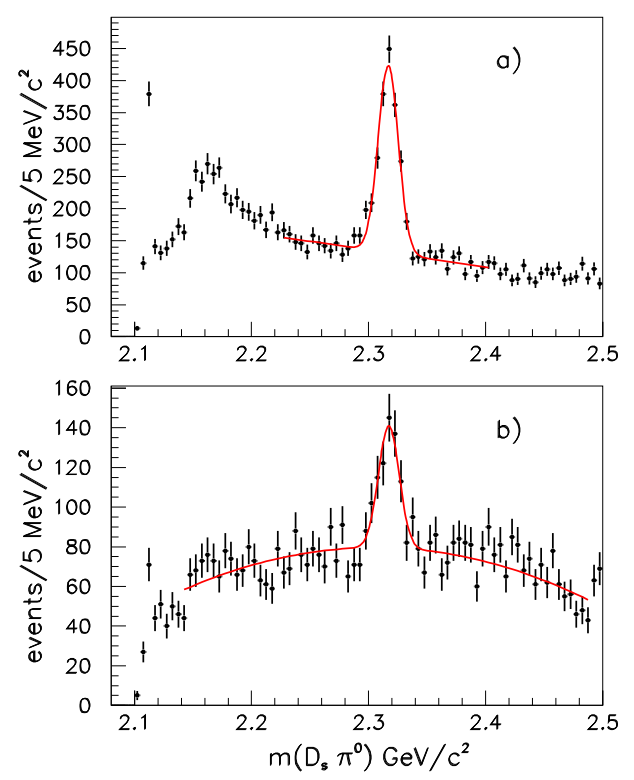

Figure 4. BaBar signal for $D_{s I}^{*}(2317) \rightarrow D_{s}^{+} \pi^{0}$ for: a) $D_{s}^{+} \rightarrow$ $K^{+} K^{-} \pi^{+}$for both $\phi \pi^{+}$and $K^{* 0} K^{+}, 1267 \pm 63$ events in the peak; and b) $D_{s}^{+} \rightarrow K^{+} K^{-} \pi^{+} \pi^{0}, 237 \pm 33$ events.

$D_{s J}^{*}(2463)$ feeds-down to $D_{s J}^{*}(2317)$ by neglecting the photon in the $D^{*}(2112) \rightarrow D_{s} \gamma$ decay. These effects were considered in all analyses. Another possibility also considered is the radiative decay $D_{s J}^{*}(2463) \rightarrow D_{s J}^{*}(2317) \gamma$.

Figures 4, 5 and 6 show BaBar, CLEO and Belle signals from their respective data set luminosities of 91, 13.5 and $87 \mathrm{fb}^{-1}$. In Table 2 we summarize their mass measurements. In all cases the width estimated by the experiments is very narrow and compatible with the detector resolution. BaBar and Belle measured the mass of $D_{s J}^{*}(2463) \sim 5 \mathrm{MeV}$ smaller than CLEO.

It is natural to interpret the $D_{s J}^{*}(2317)$ and $D_{s J}^{*}(2463)$ as the missing $J^{P} 0^{+}$and $1^{+}[c \bar{s}]$ states. Not having enough phase space to undergo the isospin conserving decay to $D K$, the decay proceeds by violating isospin, which would explain the narrowness. Several facts support this interpretation: $D_{s J}^{*}(2317)$ was not observed in the final states $D_{s} \pi^{+} \pi^{-}, D_{s} \pi^{0} \pi^{0}$ and $D_{s} \gamma$ which are forbidden for a $0^{+}$state; $D_{s J}^{*}(2463)$ decays to $D_{s} \gamma$, consequently it is not a $0^{+}$; the helicity distribution for $D_{s J}^{*}(2317) \rightarrow D_{s} \pi^{0}$ measured by BaBar is uni- 


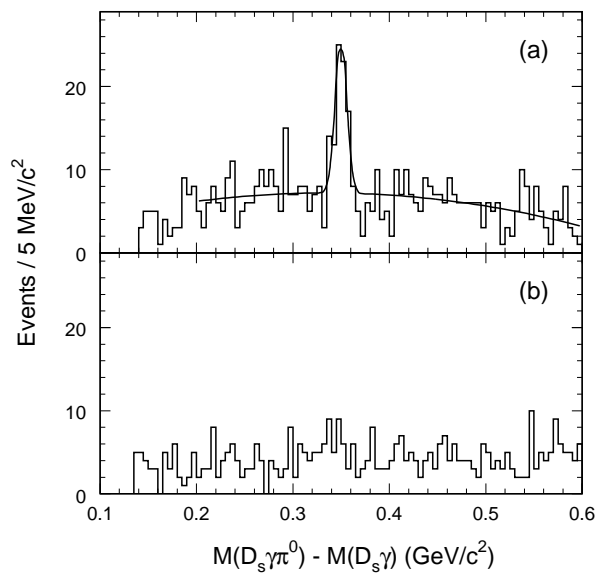

Figure 5. CLEO signal for $D_{s J}^{*}(2463)$.

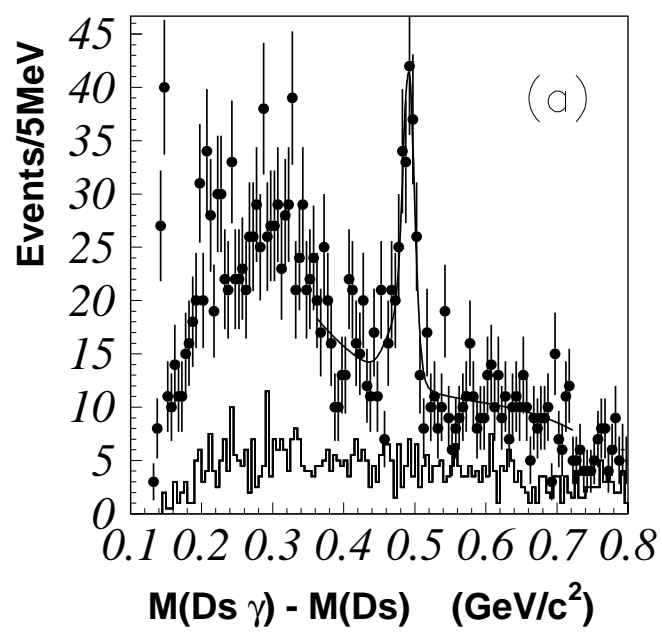

Figure 6. Belle $D_{s J}^{*}(2463)$ radiative decay.

form, consistent with $0^{+} ;^{13}$ and Belle finds the $B \rightarrow$ $D D_{s J}^{*}(2463), D_{s J}^{*}(2463) \rightarrow D_{s} \gamma$ exclusive decay helicity angle distribution consistent with $J=2$.

\subsection{Baryons}

The spectroscopy of the charm baryons is a lot more complex and less studied than the meson sector. $\mathrm{SU}(4)$ quark model multiplets are used as a guide to identify the observed states, and none of the $J^{P}$ values have been directy measured. Of the single charm ground states only the $\Omega_{0 c}^{*}\left(J^{P}=3 / 2^{+}\right)$remains undetected.

The first observation of the doubly-charmed
Table 2. Mass measurements for $D_{s J}^{*}(2317)$ and $D_{s J}^{*}(2463)$ states.

\begin{tabular}{|c|c|}
\hline & BaBar \\
\hline$M\left(D_{s J}^{*}(2317)\right) \mathrm{MeV}$ & $2316.8 \pm 0.4 \pm 0.3$ \\
\hline$M\left(D_{s J}^{*}(2317)\right)-M\left(D_{s}\right) \mathrm{MeV}$ & $348.4 \pm 0.4 \pm 0.3$ \\
\hline$M\left(D_{s J}^{*}(2463)\right) \mathrm{MeV}$ & $2457.0 \pm 1.4 \pm 3$ \\
\hline$M\left(D_{s J}^{*}(2463)\right)-M\left(D_{s}^{*}\right) \mathrm{MeV}$ & $344.6 \pm 1.2 \pm 3$ \\
\hline \hline & Belle \\
\hline$M\left(D_{s J}^{*}(2317)\right) \mathrm{MeV}$ & $2317.2 \pm 0.5 \pm 0.9$ \\
\hline$M\left(D_{s J}^{*}(2317)\right)-M\left(D_{s}\right) \mathrm{MeV}$ & $348.7 \pm 0.5 \pm 0.7$ \\
\hline$M\left(D_{s J}^{*}(2463)\right) \mathrm{MeV}$ & $2456.5 \pm 1.3 \pm 1.1$ \\
\hline$M\left(D_{s J}^{*}(2463)\right)-M\left(D_{s}^{*}\right) \mathrm{MeV}$ & $344.1 \pm 1.3 \pm 0.9$ \\
\hline \hline & CLEO \\
\hline$M\left(D_{s J}^{*}(2317)\right) \mathrm{MeV}$ & $2318.5 \pm 1.2 \pm 1.1 \pm$ \\
\hline$M\left(D_{s J}^{*}(2317)\right)-M\left(D_{s}\right) \mathrm{MeV}$ & $350.0 \pm 1.2 \pm 1.0$ \\
\hline$M\left(D_{s J}^{*}(2463)\right) \mathrm{MeV}$ & $2463.6 \pm 1.7 \pm 1.2$ \\
\hline$M\left(D_{s J}^{*}(2463)\right)-M\left(D_{s}^{*}\right) \mathrm{MeV}$ & $351.2 \pm 1.7 \pm 1.0$ \\
\hline
\end{tabular}

baryon $\Xi_{c c}^{+}$was recently published by SELEX. ${ }^{6}$ The SELEX experiment uses a $600 \mathrm{GeV}$ charged hyperon beam incident on target foils of $\mathrm{Cu}$ or diamond. In the double charm search they look for a secondary vertex of $\Lambda_{c} K^{-} \pi^{+}$within their sample of 1630 fully reconstructed $\Lambda_{c} \rightarrow p K \pi$ events. Their signal is shown in Fig. 7. It is a 6.3 standard deviation signal of 15.9 events over an estimated background of $6.1 \pm 0.5$ events. The mass is at $3519 \pm 1 \mathrm{MeV}$ identified as $[\mathrm{ccd}]^{+}$.

SELEX have pursued the search for more double charm events by requiring un extra $\pi$ track on the secondary vertex, and by imposing helicity cuts. With limited statistics SELEX presented preliminary results for 3 more [ccq] candidates. ${ }^{7}$ It is suggested that the four candidates are interpreted as the $L=0$ and $L=1[\mathrm{ccd}]^{+}$and $[\mathrm{ccu}]^{++}$.

To try and confirm these results the photoproduction experiment FOCUS have made extensive searches in their $19444 \pm 262 \Lambda_{c}$ sample. There was no evidence for a doubly charmed baryon. ${ }^{14}$

\section{Hadronic Decays}

The hadronic decays are responsible for the not so well understood large differences in the lifetimes between the charm hadrons (Fig. 1). The leptonic and semileptonic represent just a small fraction of the total charm decay width. In the semileptonic decays, for example, the hadronic complexity can be isolated in measured form factors. As expected for spectators diagrams, the semileptonic widths for the various charm hadrons are comparable. The hadronic 


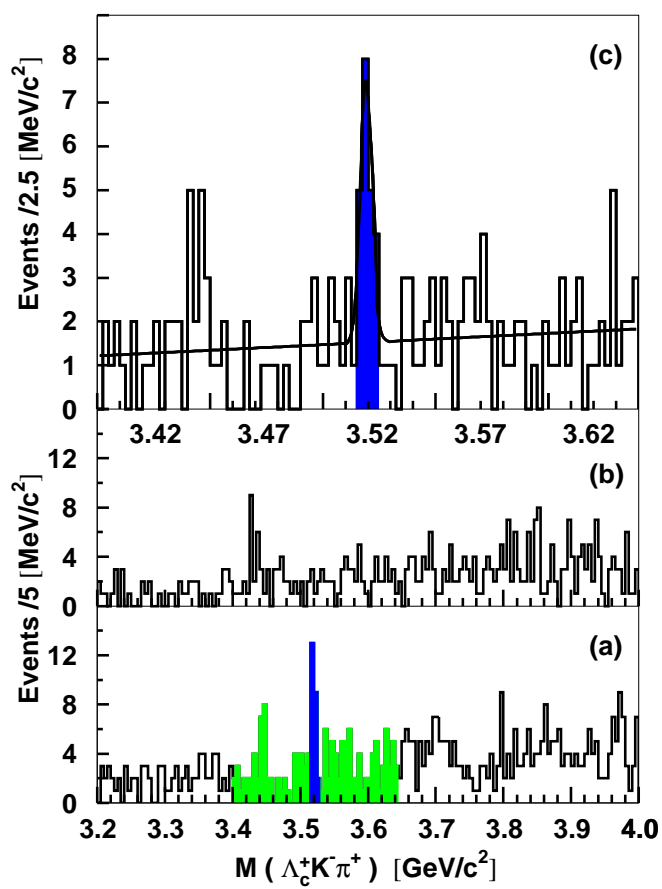

Figure 7. (a) The $\Lambda_{c} K^{-} \pi^{+}$mass distribution in $5 \mathrm{MeV}$ bins. The shaded region $3.400-3.640 \mathrm{GeV}$ contains the signal peak and is shown in more detail in (c). (b) The wrong-sign combination. (c) The signal (shaded) region (22 events) and sideband mass regions with 162 total events in $2.5 \mathrm{MeV}$ bins.

decays are much more complex and largely influenced by non-perturbative QCD effects. It has been known for a long time that the final-state interactions (FSI) can obscure the interpretation of the results and should be better understood. On the other hand, the hadronic decays are also responsible for the large diversity and rich resonant substructure observed in charm.

The simplest case are the two-body decays. It has been useful for studying final-state interactions and relative decay mechanisms, but gives no information on resonant substructure. For 3 or more bodies in the final state, generally a full amplitude analysis is desired to retrieve complete information on intermediate resonant processes and possible decay mechanisms. This can become too complex to be feasible, but in the 3-body decays involving only scalar/pseudoscalar particles there are only two degrees of freedom.

Next I summarize recent 3-body decay amplitude analyses followed by results on two, four and five bodies in the final state.

\subsection{Three-body Decays}

All results from this session are 3-body full coherent Dalitz plot analyses involving only pseudoscalar particles; $P \rightarrow P_{1} P_{2} P_{3}$. The Dalitz plot is the scatter plot of $s_{12} \times s_{13}{ }^{a}$ and it is proportional to squared decay amplitude, $\left|\mathcal{H}\left(s_{12}, s_{13}\right)\right|^{2}$. $\mathcal{H}$ is written as a coherent sum of all possible resonant intermediate contributions and a non-resonant term:

$$
\begin{array}{r}
\mathcal{H}\left(s_{12}, s_{13}\right)=\sum a_{i} \mathcal{A}_{i}\left(s_{12}, s_{13}, \vec{\alpha}_{i}\right) e^{i \delta_{i}} \\
+a_{N R} \mathcal{A}_{N R} e^{i \delta_{N R}}
\end{array}
$$

where the sum runs over the $i$ possible resonant states; $a_{i, N R}$ are the relative contributions; $\delta$ are relative phases that accommodate the final-state interaction effects; and $\mathcal{A}$ is the resonant amplitude that depend on parameters $\vec{\alpha}$.

The parameters $a_{i, N R}$ and $\delta$ are extracted from likelihood fits to the data. Usually, the resonances are represented by Breit-Wigners in which case $\vec{\alpha}$ are its mass and width, that may or may not be free parameters of the fits.

There are two limitations on this kind of analysis. First one needs to impose a parametrization for the resonances. Relativistic Breit-Wigner forms are widely used but there are known limitations particularly for broad states near threshold. Second, as the number of states allowed becomes large, the interpretation is not straightforward. The challenge is to define clear quality criteria that, along with physics insights, could guide the analysis.

Nevertheless, the amplitude analysis is a powerful tool to investigate both charm decays and light meson spectroscopy, specially in the case of scalars that seem to be favored in charm decays. It is important to compare and understand the differences between hadron scattering and charm decay environments, for which final-state interactions play an important role.

\subsection{1. $E 791-D^{+} \rightarrow \pi^{-} \pi^{+} \pi^{+}$}

In a full Dalitz plot analysis of the decay $D^{+} \rightarrow$ $\pi^{-} \pi^{+} \pi^{+},{ }^{15}$ E791 found a large contribution of a low mass broad scalar state, that the authors identified with the $\sigma(500)$ meson. Using a standard relativistic

${ }^{a_{s_{12}}=m_{12}^{2} \text { and } s_{13}=m_{13}^{2}}$ are the squared invariant masses of the pairs of particles $P_{1}$ and $P_{2}$, and $P_{2}$ and $P_{3}$ respectively. 
Breit-Wigner parametrization, they measured $m_{0}=$ $478_{-23}^{+24} \pm 17 \mathrm{MeV}$ and $\Gamma_{0}=324_{-40}^{+42} \pm 21 \mathrm{MeV}$. Using only established resonances, E791 was unable to achieve a satisfactory solution, there was a clear mismatch at low $\pi^{+} \pi^{-}$invariant mass. Likelihood difference tests determined that there is a statistically significant preference seen in the data for a scalar resonance model if compared to a vector, tensor or even just a phaseless structure hypothesis.

The $\sigma(500)$ has a fundamental role in several spontaneous chiral symmetry breaking models and has been actively searched for in scattering experiments but no direct evidence for its existence has been established. The E791 result raised a series of debates on the subject. The strongest criticism, has been the fact that in the Dalitz plot analysis the resonance form is imposed. Moreover, the $\sigma(500)$ is a wide scalar state near threshold, conditions not favored for a Breit-Wigner width parametrization.

To feed in more information on the subject, E791 have applied the recently proposed Amplitude Difference (AD) method ${ }^{16}$ to the $D^{+} \rightarrow \pi^{-} \pi^{+} \pi^{+}$decay. ${ }^{17}$ They perform a direct and model-independent measurement of the phase motion of the amplitude at the low $\pi^{+} \pi^{-}$mass region. Their preliminary results show a strong phase variation compatible with the isoscalar $\sigma(500)$ meson.

The applicability of the AD method requires a clean region of the Dalitz plot where the only contributions are the generic amplitude under study crossing with a well established resonance, represented by a Breit-Wigner. In the $D^{+} \rightarrow \pi^{-} \pi^{+} \pi^{+}$, the only region that fulfill this condition is the region pictured in Fig. 8 where the low mass region in $s_{13}$ crosses with the $f_{2}(1270)$ in $s_{12}$. Under these circumstances, the amplitude is written as:

$$
\begin{gathered}
\mathcal{A}\left(s_{12}, s_{13}\right)=a_{R} \mathcal{B W}_{f_{2}}\left(s_{12}, m_{0}, \Gamma_{0}\right) \mathcal{M}\left(s_{12}, s_{13}\right) \\
+\left(a_{s} /\left(p^{*} / \sqrt{s_{13}}\right)\right) \sin \delta\left(s_{13}\right) e^{i\left(\delta\left(s_{13}\right)+\gamma\right)},
\end{gathered}
$$

the first term on the right-hand-side represents the well established $f_{2}(1270)$ Breit-Wigner resonance of mass $m_{0}$, width $\Gamma_{0}$ and angular distribution $\mathcal{M}$, and the second term represents the generic amplitude under study. $\left(p^{*} / \sqrt{s_{13}}\right)$ is a kinematic term to make this description compatible with the usual $\pi \pi$ scattering formulation. $\delta\left(s_{13}\right)$ is the desired phase motion, $a_{R}$ and $a_{s}$ are the relative contributions and $\gamma$ is the final-state interaction phase difference also

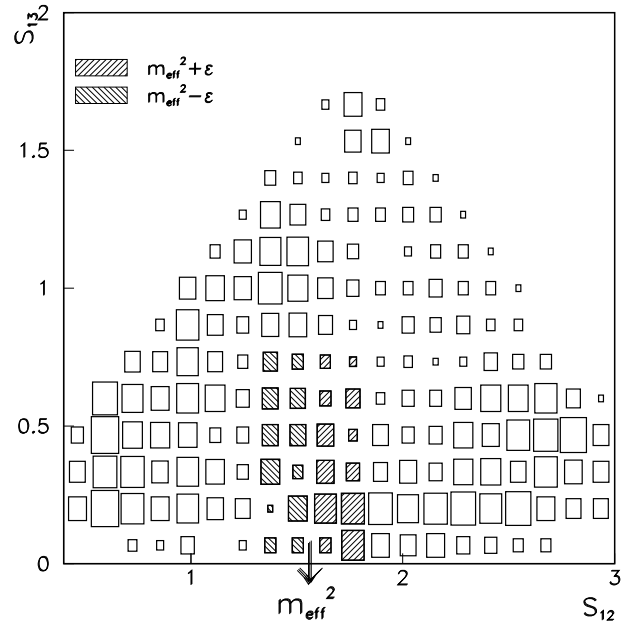

Figure 8. E791 $D^{+} \rightarrow \pi^{-} \pi^{+} \pi^{+}$folded Dalitz plot. Dashed region used in the $\mathrm{AD}$ method.

present in the full Dalitz plot analysis.

The $\mathrm{AD}$ method consists of making the amplitude-squared difference $\Delta|\mathcal{A}|^{2} \equiv \mid \mathcal{A}\left(m_{0}^{2}+\right.$ $\left.\epsilon, s_{13}\right)\left.\right|^{2}-\left|\mathcal{A}\left(m_{0}^{2}-\epsilon, s_{13}\right)\right|^{2}$, that takes a very simple functional form $s_{13}$ if the resonance used as the probe is symmetrical with respect to $m_{0}$. In the case that the probe is not a narrow scalar resonance, the application of the $\mathrm{AD}$ method requires some approximations. Both the angular distribution and the mass dependent width of $f_{2}(1270)$ affects the symmetry with respect to $m_{0}^{2}=1.61 \mathrm{GeV}^{2}$. The two approximations used in the E791 application of the method are that $\mathcal{M}\left(s_{12}, s_{13}\right) \sim \mathcal{M}\left(s_{13}\right)$, and instead of the nominal mass they used an effective value $m_{\text {eff }}^{2}=1.535 \mathrm{GeV}^{2}$. In this case the integrated amplitude-squared difference is:

$$
\begin{array}{r}
\Delta|\mathcal{A}|^{2} \sim-\mathcal{C}\left[\sin \left(2 \delta\left(s_{13}\right)+\gamma\right)-\sin \gamma\right] \\
\overline{\mathcal{M}}_{f_{2}(1270)}\left(s_{13}\right) /\left(p^{*} / \sqrt{s_{13}}\right)
\end{array}
$$

where $\mathcal{C}$ is a constant to be determined experimentally. The difference on the left-hand-side of this equation is taken from the two dashed regions of Fig. 8 where $\epsilon=0.26 \mathrm{GeV}^{2}$. The amplitude squared difference divided by the angular distribution and phase space factor is shown in Fig. 9a. Notice that the 6 th bin has a large error, this is due to a singularity in $\mathcal{M}$. This bin was not further used in the analysis. From the above Eq. (3) it is clear that any variation observed in Fig. 9a reflects a variation in $\delta\left(s_{13}\right)$. 


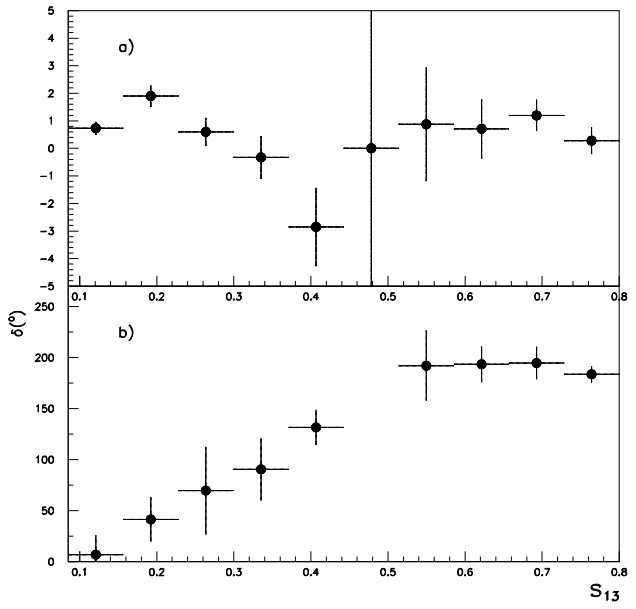

Figure 9. E791 amplitude difference: a) difference of the two shaded areas of Fig. 8 divided by the angular distribution and the phase space factor; and b) phase motion for the low $\pi^{+} \pi^{-}$ invariant mass region.

The maximum and minimum values of $\Delta \mathcal{A} / \mathcal{M}$ (in the present case bins 2 and 5 of Fig. 9a) provide two equations that are solved for $\mathcal{C}$ and $\gamma$. Eq. (3) is then inverted for $\delta\left(s_{13}\right)$. There are ambiguities that arise due to the arcsin operations and they are solved by imposing that $\delta\left(s_{13}\right)$ be a smooth increasing function of $s_{13}$. Finally, Fig. 9b shows the phase motion of the low $\pi^{+} \pi^{-}$mass region obtained with the $\mathrm{AD}$ method. This strong phase motion is compatible with the full Breit-Wigner phase variation confirming the result obtained in the full Dalitz plot analysis. The measured $\gamma=2.78 \pm 0.33 \mathrm{rad}$ is also compatible with previous results, $\gamma_{\text {Dalitz }}=2.59 \pm 0.19 \mathrm{rad}$.

\subsection{2. $C L E O-D^{*+} \rightarrow D^{0} \pi^{+} ; D^{0} \rightarrow K_{s} \pi^{-} \pi^{+}$}

CLEO has reported on the Dalitz plot analysis of the channel $D^{*+} \rightarrow D^{0} \pi^{+} ; D^{0} \rightarrow K_{s} \pi^{-} \pi^{+} .18$ Their Dalitz plot are shown in Fig. 10d. The sample is of $\sim 5300$ events over a small $2 \%$ background. The $D^{*}$-tag is necessary to identify the favored decays $\left(\bar{K}^{0} \pi^{-} \pi^{+}\right)$and the doubly-Cabibbo-suppressed or mixing contributions $\left(K^{0} \pi^{+} \pi^{-}\right)$. This analysis can be very complex because there are many possible resonant states. CLEO has considered up to 20 contributions: a non-resonant; $10\left[\pi^{+} \pi^{-}\right] ; 7\left[K_{s} \pi^{-}\right]$favored and $2\left[K_{s} \pi^{+}\right]$suppressed states. Their final result is listed in Table 3 and the model is compared to
Table 3. CLEO - $D^{*+} \rightarrow D^{0} \pi^{+} ; D^{0} \rightarrow K_{s} \pi^{-} \pi^{+}$. I listed only statistical errors.

\begin{tabular}{|c|c|c|}
\hline Component & Phase & Fit fraction\% \\
\hline$K^{*}(892)^{+} \pi^{-}$ & $321 \pm 10$ & $0.34 \pm 0.13$ \\
\hline $\bar{K}^{0} \rho$ & $0($ fixed $)$ & $26.4 \pm 0.9$ \\
\hline $\bar{K}^{0} w$ & $114 \pm 7$ & $0.72 \pm 1.8$ \\
\hline$K^{*}(892)^{-} \pi^{+}$ & $150 \pm 2$ & $65.7 \pm 1.3$ \\
\hline $\bar{K}^{0} f_{0}(980)$ & $188 \pm 4$ & $4.3 \pm 0.5$ \\
\hline $\bar{K}^{0} f_{2}(1270)$ & $308 \pm 12$ & $0.27 \pm 0.15$ \\
\hline $\bar{K}^{0} f_{0}(1370)$ & $85 \pm 4$ & $9.9 \pm 1.1$ \\
\hline$K_{0}^{*}(1430) \pi^{+}$ & $3 \pm 4$ & $7.3 \pm 0.7$ \\
\hline$K_{2}^{*}(1430) \pi^{+}$ & $155 \pm 7$ & $1.1 \pm 0.2$ \\
\hline$K^{*}(1680) \pi^{+}$ & $174 \pm 6$ & $2.2 \pm 0.4$ \\
\hline$N . R$. & $160 \pm 11$ & $0.9 \pm 0.4$ \\
\hline
\end{tabular}

data in Fig. 10. One can see from Fig. 10b a discrepancy in the low $\pi^{+} \pi^{-}$region. They have opted to not include the $\sigma(500)$ in their final result but when they include it as a regular Breit-Wigner, they observe a sizable fraction of $f_{\sigma}=0.57 \pm 0.13$ and measure $m_{\sigma}=513 \pm 32 \mathrm{MeV}$ and $\Gamma_{\sigma}=335 \pm 67 \mathrm{MeV}$, values compatible with those measured by E791. They do not mention the improvement that the inclusion of the $\sigma(500)$ does to the fit quality.

CLEO sees a small but statistically significant contribution of 5.5 standard deviations from the doubly-Cabibbo-suppressed or $D^{0}-\bar{D}^{0}$ mixing $K^{*}(892)^{+} \pi^{-}$intermediate state. They report for the first time the relative branching ratio, $B R\left(D^{0} \rightarrow\right.$ $\left.K^{*}(892)^{+} \pi^{-}\right) / B R\left(D^{0} \rightarrow K^{*}(892)^{-} \pi^{+}\right)=(0.5 \pm$ $\left.0.2_{-0.1-0.1}^{+0.5+0.4}\right) \%$. Comparing the phases of the two $K^{*}(892) \pi$ channels they observe no CP-violating effects.

\subsection{3. $C L E O-D^{*+} \rightarrow D^{0} \pi^{+} ; D^{0} \rightarrow \pi^{0} \pi^{-} \pi^{+}$}

CLEO have preliminary results on the Dalitz plot analysis of the decay $D^{*+} \rightarrow D^{0} \pi^{+} ; D^{0} \rightarrow$ $\pi^{0} \pi^{-} \pi^{+} .19$ One of the strongest motivations of this analysis is the search for the $\sigma(500)$. In principle, there is no impeachment for the existence of this intermediate channel. The charged intermediate modes, $\rho^{+} \pi^{-}$and $\rho^{-} \pi^{+}$, are possible via spectator diagrams and the neutral modes such as $\rho^{0} \pi^{0}$, $f_{0}(980) \pi^{0}$ or $\sigma(500) \pi^{0}$ are produced in internal $W$ emission or $W$-exchange type diagrams. The $D^{*}$-tag is required to distinguish $D^{0}$ from $\bar{D}^{0}$ and to search for the manifestation of CP-violation. Their final signal sample has $\sim 1100$ events over a $\sim 18 \%$ background contamination. The phases and fractions for their preferred model are listed in Table 4 . The vec- 

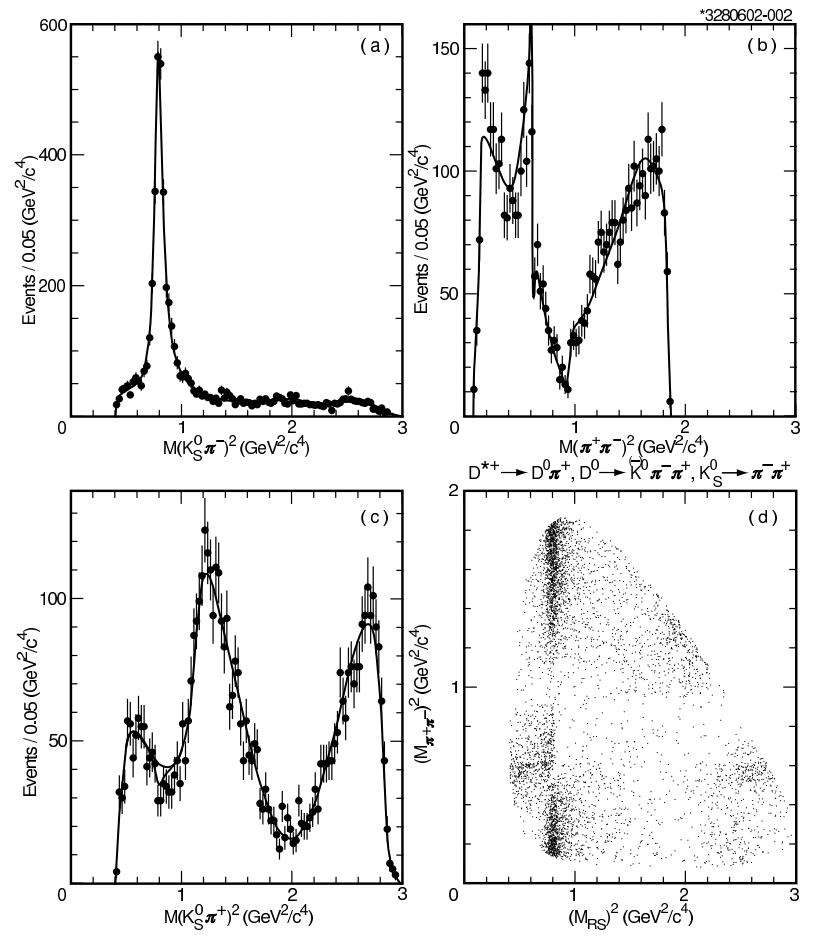

Figure 10. CLEO - $D^{0} \rightarrow K_{s} \pi^{-} \pi^{+}$projections compared to the best fit, and the Dalitz plot.

Table 4. CLEO - $D^{*+} \rightarrow D^{0} \pi^{+} ; D^{0} \rightarrow \pi^{0} \pi^{-} \pi^{+}$.

\begin{tabular}{|c|c|c|}
\hline Mode & Phase $\left({ }^{o}\right)$ & Fit fraction\% \\
\hline$\rho^{+}$ & $0\left(\right.$ fixed $\left.^{+}\right)$ & $76.5 \pm 1.8 \pm 4.8$ \\
\hline$\rho^{0}$ & $10 \pm 3 \pm 3$ & $23.9 \pm 1.8 \pm 4.6$ \\
\hline$\rho^{-}$ & $-4 \pm 3 \pm 4$ & $32.3 \pm 2.1 \pm 2.2$ \\
\hline N.R. & $77 \pm 8 \pm 11$ & $2.7 \pm 0.9 \pm 1.7$ \\
\hline
\end{tabular}

tor $\rho \pi$ contributions dominates. There is a small non-resonant component and no scalar resonant is needed to explain the data. No sign of CP-violation is observed.

$$
\begin{array}{ll}
\text { 3.1.4. } & \text { BaBar - } D^{*+} \rightarrow D^{0} \pi^{+} ; D^{0} \rightarrow \\
& K_{s} H H,\left(H=K^{ \pm}, \pi^{ \pm}\right)
\end{array}
$$

BaBar have nice clean samples of four 3-body decays containing one neutral $K_{s}$ in the final state, combined with charged kaons or pions. ${ }^{20}$ They have preliminary Dalitz plot analysis results for the channels $K^{0} K^{-} \pi^{+}, \bar{K}^{0} K^{-} \pi^{+}$and $K^{0} K^{-} K^{+}$from $22 \mathrm{fb}^{-1}$ with event samples of 1008, 659 and 1957 events respectively and small background contamination of the order of $\sim 4 \%$ or less. They have an amazing $K_{s} \pi^{-} \pi^{+}$sample of over 15000 events, but no result have so far been released. The difficulty in all these
Table 5. BaBar $-D^{*+} \rightarrow D^{0} \pi^{+} ; D^{0} \rightarrow K^{0} K^{-} \pi^{+}$.

\begin{tabular}{|c|c|c|}
\hline Component & Phase & Fit fraction\% \\
\hline $\bar{K}^{0}(1430) K^{0}$ & $52 \pm 27$ & $4.8 \pm 1.4 \pm 1.6$ \\
\hline $\bar{K}^{0}(892) K^{0}$ & $175 \pm 22$ & $0.8 \pm 0.5 \pm 0.1$ \\
\hline $\bar{K}^{* 0}(1680) K^{0}$ & $-169 \pm 16$ & $6.9 \pm 1.2 \pm 1.0$ \\
\hline $\bar{K}_{2}^{*}(1430) K^{0}$ & $51 \pm 18$ & $2.0 \pm 0.6 \pm 0.1$ \\
\hline$K^{*+}(1430) K^{-}$ & $-41 \pm 25$ & $13.3 \pm 3.5 \pm 3.9$ \\
\hline$K^{*+}(892) K^{-}$ & $0($ fixed $)$ & $63.6 \pm 5.1 \pm 2.6$ \\
\hline$K^{*+}(1680) K^{-}$ & $-178 \pm 10$ & $15.6 \pm 3.0 \pm 1.4$ \\
\hline$K_{2}^{*+}(1430) K^{-}$ & $-52 \pm 7$ & $13.8 \pm 2.6 \pm 7.9$ \\
\hline$a_{0}^{-}(980) \pi^{+}$ & $-100 \pm 13$ & $2.9 \pm 2.3 \pm 0.7$ \\
\hline$a_{0}^{-}(1450) \pi^{+}$ & $31 \pm 16$ & $3.1 \pm 1.9 \pm 0.9$ \\
\hline$a_{2}^{-}(1310) \pi^{+}$ & $-149 \pm 27$ & $0.7 \pm 0.4 \pm 0.1$ \\
\hline$N R$ & $-136 \pm 23$ & $2.3 \pm 0.5 \pm 5.6$ \\
\hline
\end{tabular}

analyses is that there are too many possible resonant modes, despite the fact that the Dalitz plots present quite strong contributions of just a few, mostly vector resonances. If many possible states are allowed in the fit, it is quite probable that several different mathematical solutions fit the data with comparable confidence level. The situation gets even more critical because some of the possible resonances are controversial with poorly measured parameters.

In Table 5 we list the $K^{0} K^{-} \pi^{+}$proposed solution. There is a very large contribution $(\sim 63 \%)$ from $K^{*}(892)^{+} K^{-}$and eleven more states are allowed. A good $\chi^{2} / D O F=46 / 44$ is obtained, but with a large destructive interference, with the fractions summing to $130 \pm 8 \%$. In this channel the authors do not find a significant contribution of the $\kappa$ meson. ${ }^{21}$ In the case of the channel $K^{0} K^{+} \pi^{-}$listed in Table 6 , eleven intermediate states are kept with fractions that sum to $144 \pm 37 \%$. Notice the large NR contribution, unusual for charm decays. The authors point out that if the NR is removed from the fit, it converges, increasing the $K_{0}^{*}(1430)^{-} K^{+}$to $26 \%$ and decreasing the $a_{0}^{+}(980) \pi^{-}$to $5 \%$. The two solutions, with and without NR, have approximately the same quality.

Finally BaBar reports on the $3 K$ mode where again the preferred solution presents a large destructive interference, see Table 7 . Notice that in this solution the contribution of the poorly measured and near-the-threshold contribution of the $a_{0}(980)$ is dominant, much larger than that of the $\bar{K}^{0} \phi$.

\subsubsection{E791 - $D^{+} \rightarrow K^{-} \pi^{+} \pi^{+}$}

The Cabibbo-favored $D^{+} \rightarrow K^{-} \pi^{+} \pi^{+}$was one of the first modes to have a full amplitude analysis done. ${ }^{21}$ 
Table 6. BaBar - $D^{*+} \rightarrow D^{0} \pi^{+} ; D^{0} \rightarrow \bar{K}^{0} K^{+} \pi^{-}$.

\begin{tabular}{|c|c|c|}
\hline Component & Phase & Fit fraction\% \\
\hline$K^{* 0}(1430) \bar{K}^{0}$ & $-38 \pm 22$ & $26.0 \pm 16.1 \pm 3.3$ \\
\hline$K^{* 0}(892) \bar{K}^{0}$ & $-126 \pm 19$ & $2.8 \pm 1.4 \pm 0.5$ \\
\hline$K^{* 0}(1680) \bar{K}^{0}$ & $161 \pm 9$ & $15.2 \pm 11.9 \pm 0.5$ \\
\hline$K_{2}^{* 0}(1430) \bar{K}^{0}$ & $53 \pm 38$ & $1.7 \pm 2.5 \pm 0.2$ \\
\hline$K^{*-}(1430) K^{+}$ & $-142 \pm 115$ & $2.4 \pm 8.2 \pm 1.0$ \\
\hline$K^{*-}(892) K^{+}$ & $0($ fixed $)$ & $35.6 \pm 7.7 \pm 2.4$ \\
\hline$K^{*-}(1680) K^{+}$ & $124 \pm 27$ & $5.1 \pm 5.7 \pm 1.1$ \\
\hline$K_{2}^{*-}(1430) K^{+}$ & $-26 \pm 38$ & $1.0 \pm 1.0 \pm 0.2$ \\
\hline$a_{0}^{+}(980) \pi^{-}$ & $-160 \pm 42$ & $15.1 \pm 12.5 \pm 0.6$ \\
\hline$a_{0}^{+}(1450) \pi^{-}$ & $148 \pm 25$ & $2.2 \pm 2.7 \pm 1.2$ \\
\hline$N R$ & $-172 \pm 13$ & $36.6 \pm 25.8 \pm 2.7$ \\
\hline
\end{tabular}

Table 7. BaBar $-D^{*+} \rightarrow D^{0} \pi^{+} ; D^{0} \rightarrow \bar{K}^{0} K^{+} K^{-}$.

\begin{tabular}{|c|c|c|}
\hline Component & Phase & Fit fraction\% \\
\hline $\bar{K}^{0} \phi$ & $0($ fixed $)$ & $45.4 \pm 1.6 \pm 1.0$ \\
\hline $\bar{K}^{0} a_{0}^{0}(980)$ & $109 \pm 5$ & $60.9 \pm 7.5 \pm 13.3$ \\
\hline $\bar{K}^{0} f_{0}(980)$ & $-161 \pm 14$ & $12.2 \pm 3.1 \pm 8.6$ \\
\hline$a_{0}^{+}(980) K^{-}$ & $-53 \pm 4$ & $34.3 \pm 3.2 \pm 6.8$ \\
\hline$a_{0}^{-}(980) K^{+}$ & $-13 \pm 15$ & $3.2 \pm 1.9 \pm 0.5$ \\
\hline$N R$ & $40 \pm 44$ & $0.4 \pm 0.3 \pm 0.8$ \\
\hline \multicolumn{2}{|l}{}
\end{tabular}

E691 and E687 ${ }^{22}$ with large samples and using only well established resonances observed a large interfering pattern, large NR contributions in bad quality fits. E791, with a large sample of over 15000 events and a small background, $6 \%$, first tried some more sophisticated models for the NR contribution, but were unable to fit the data reasonably. They could only reach a good confidence level when they allowed for the possibility of a new resonant state. Doing extensive fit quality and consistency tests they concluded that the new contribution is a scalar, $\kappa$, with mass $797 \pm 19 \pm 43 \mathrm{MeV}$ and width $410 \pm 43 \pm 87 \mathrm{MeV}$. Their result with and without the $\kappa$ are compared in Table 8 . The two solutions are very different. With the inclusion of the new state the NR contribution drops from $90 \%$ to $13 \%$ following the general trend that charm decays tend to be quasi-two-body. The large destructive interfering pattern is no longer present and the $\chi^{2} / 63$ went from 2.7 to 0.75 .

Favoring the new E791 solution is the fit quality and the simplicity in a high statistics sample. The problem with this solution is that the $\kappa$ is not a well established resonance, it was not observed in $K \pi$ scattering and moreover, it is a wide scalar close to the threshold.

E791 have presented preliminary studies comparing the phase of the S-wave component of the
Table 8. Results without the $\kappa$ and with the $\kappa$.

\begin{tabular}{|c|cc|}
\hline & \multicolumn{2}{|c|}{ No $\kappa$} \\
\hline Mode & Fraction (\%) & Phase \\
\hline NR & $90.9 \pm 2.6$ & $0^{\circ}$ (fixed) \\
$\kappa \pi^{+}$ & - & - \\
$\bar{K}^{*}(892) \pi^{+}$ & $13.8 \pm 0.5$ & $(54 \pm 2)^{\circ}$ \\
$\bar{K}_{0}^{*}(1430) \pi^{+}$ & $30.6 \pm 1.6$ & $(54 \pm 2)^{\circ}$ \\
$\bar{K}_{2}^{*}(1430) \pi^{+}$ & $0.4 \pm 0.1$ & $(33 \pm 8)^{\circ}$ \\
$\bar{K}^{*}(1680) \pi^{+}$ & $3.2 \pm 0.3$ & $(66 \pm 3)^{\circ}$ \\
\hline & \multicolumn{2}{|c|}{ With $\kappa$} \\
\hline NR & $13.0 \pm 5.8 \pm 4.4$ & $(-11 \pm 14 \pm 8)^{\circ}$ \\
$\kappa \pi^{+}$ & $47.8 \pm 12.1 \pm 5.3$ & $(187 \pm 8 \pm 18)^{\circ}$ \\
$\bar{K}^{*}(892) \pi^{+}$ & $12.3 \pm 1.0 \pm 0.9$ & $0^{\circ}($ fixed) \\
$\bar{K}_{0}^{*}(1430) \pi^{+}$ & $12.5 \pm 1.4 \pm 0.5$ & $(48 \pm 7 \pm 10)^{\circ}$ \\
$\bar{K}_{2}^{*}(1430) \pi^{+}$ & $0.5 \pm 0.1 \pm 0.2$ & $(-54 \pm 8 \pm 7)^{\circ}$ \\
$\bar{K}^{*}(1680) \pi^{+}$ & $2.5 \pm 0.7 \pm 0.3$ & $(28 \pm 13 \pm 15)^{\circ}$ \\
\hline
\end{tabular}

$D^{+} \rightarrow K^{-} \pi^{+} \pi^{+}$decay amplitude with the phase observed in $K \pi$ scattering experiments. ${ }^{23}$ In the scattering experiment LASS, ${ }^{24}$ a slow phase motion is observed for the scalar partial wave at the low $K \pi$ invariant mass region, not compatible with the variation characteristics of a resonance. The S-wave amplitude used by LASS with a phase space adapted for the $D$ decay is given by:

$$
\begin{aligned}
\mathcal{A}_{S}= & \frac{m_{12}}{p_{12}^{*}} \sin \delta_{B} e^{i \delta_{B}} \\
& +e^{2 i \delta_{B}} \frac{\left(m_{0}^{2} / p_{12}^{* 0}\right) \Gamma_{0}}{m_{0}^{2}-m_{12}^{2}-i m_{0} \Gamma\left(m_{12}\right)} \\
& +(2 \leftrightarrow 3)
\end{aligned}
$$

with $\Gamma(m)=\left(m_{0} / m\right)\left(p^{*} / p^{* 0}\right) \Gamma_{0}$ and the NR term has the effective range form:

$$
\cot \delta_{B}=\frac{1}{a p^{*}}+\frac{1}{2} b p^{*}
$$

The above S-wave parametrization was used to fit E791 data and the measured parameters are: $a=$ $4.58 \pm 0.33 \mathrm{c} / \mathrm{GeV}, b=-2.94 \pm 0.43 \mathrm{c} / \mathrm{GeV}$, to be compared with the values $a=1.95 \pm 0.09 \mathrm{c} / \mathrm{GeV}$ and $b=1.76 \pm 0.36 \mathrm{c} / \mathrm{GeV}$ from LASS. Figure 11 compares the S-wave phase for the $\kappa$ Breit-Wigner solution with the scattering parametrization, with $a$ and $b$ fixed at the values measured by LASS and measured by E791. From the plot we see that the scalar sector at low $K \pi$ mass in $D^{+} \rightarrow K^{-} \pi^{+} \pi^{+}$ prefers a rapidly varying phase, consistent with the previous indication of the $\kappa$ state. 


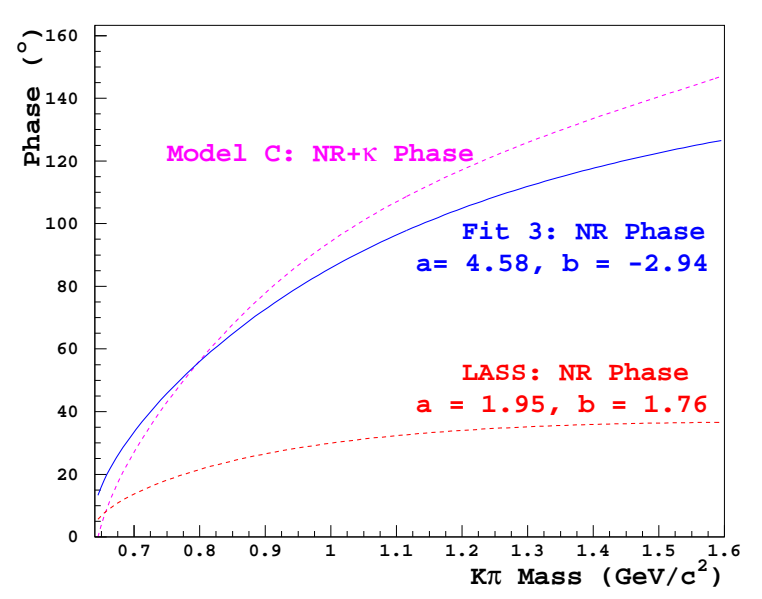

Figure 11. E791 $D^{+} \rightarrow K^{-} \pi^{+} \pi^{+}$phase of the scalar amplitude as a function of $K \pi$ invariant mass. Model C: from the Dalitz plot fit of E791 data using a flat NR plus a $\kappa$ resonance parametrized as a Breit-Wigner. Fit 3: NR alone using the LASS parametrization allowing $a$ and $b$ parameters to float. LASS: for comparison the scalar amplitude as measured by LASS for $K \pi$ scattering.

Table 9. CLEO preliminary relative branching ratios.

\begin{tabular}{|c|c|c|}
\hline$D^{+}$ & CLEO (\%) & PDG (\%) \\
\hline$\frac{\Gamma\left(\pi^{+} \pi^{0}\right)}{\Gamma\left(K^{-} \pi^{+} \pi^{+}\right)}$ & $1.44 \pm 0.19 \pm 0.10$ & $2.08 \pm 0.6 \pm 0.5$ \\
\hline$\frac{\Gamma\left(K^{+} K_{s}\right)}{\Gamma\left(\pi^{+} K_{s}\right)}$ & $18.92 \pm 1.55 \pm 0.73$ & $28.3 \pm 3.5$ \\
\hline$\frac{\Gamma\left(K^{+} \pi^{0}\right)}{\Gamma\left(K^{-} \pi^{+} \pi^{+}\right)}$ & $0.29 \pm 0.18 \pm 0.09$ & -- \\
\hline
\end{tabular}

\subsection{Decays with 2 Bodies or More than 3 Bodies}

FOCUS ${ }^{25}{ }^{\mathrm{CLEO}^{26}}$ and $\mathrm{CDF}^{18}$ have new numbers on some singly- and doubly-Cabibbo-suppressed decays.

With an integrated luminosity of $13.7 \mathrm{fb}^{-1}$, CLEO have new numbers for $D^{+} \rightarrow \pi^{+} \pi^{0}, D^{+} \rightarrow$ $K^{+} K_{s}$ and $D^{+} \rightarrow K^{+} \pi^{0}$. Figure 12 shows the signals and in Table 9 the relative branching ratios are compared to their previous results. With the PDG value for the $D^{+} \rightarrow K^{-} \pi^{+} \pi^{+}$branching ratio, they set the limit $<4.2 \times 10^{-4}$ at $90 \%$ CL for the $D^{+} \rightarrow K^{+} \pi^{0}$ branching ratio.

CDF has preliminary results on $D^{0} \rightarrow \pi^{+} \pi^{-}$ and $D^{0} \rightarrow K^{+} K^{-}$from $65 \mathrm{pb}^{-1}$ integrated luminosity. They use the $D^{*}$-tag to clean their signals shown in Fig. 13. In Table 10, CDF and FOCUS measurements for the relative branching ratios for these channels are compared to previous measurements. The normalizing signals, $D^{0} \rightarrow K^{-} \pi^{+}$, are of $94560 \pm 340$ and $105030 \pm 372$ events respectively
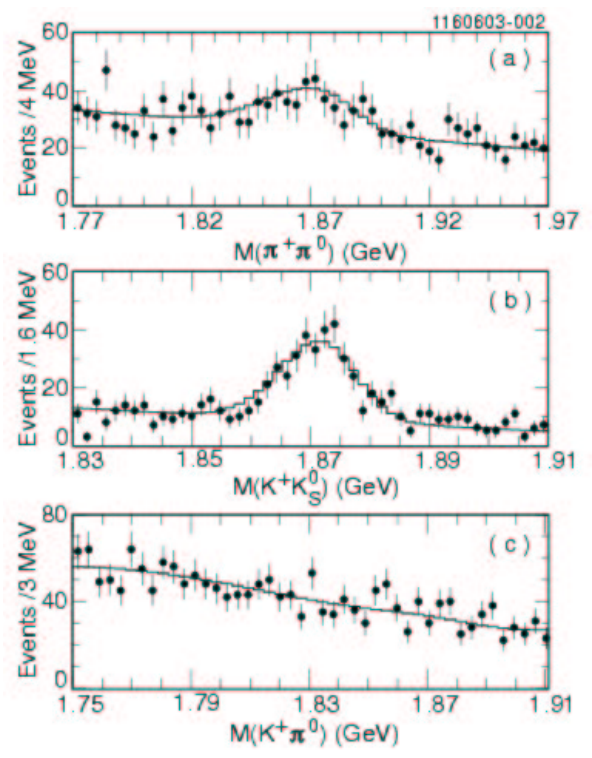

Figure 12. CLEO $D^{+} \rightarrow \pi^{+} \pi^{0}, K^{+} K_{s}, K^{+} \pi^{0}$.
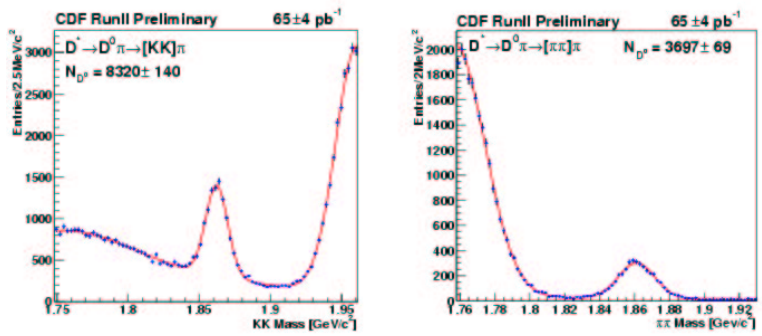

Figure 13. CDF preliminary $D^{0} \rightarrow \pi^{+} \pi^{-}$and $D^{0} \rightarrow K^{+} K^{-}$.

for CDF and FOCUS. In the $\pi^{+} \pi^{-}$mode, the small errors in the $\mathrm{CDF}$ results are a demonstration of the large potential capability they have to do charm physics.

To get insight into the importance of the finalstate interactions on charm decays, the $D \rightarrow \pi \pi$ and $D \rightarrow K K$ decay amplitudes are expressed in terms of isospin amplitudes. The $\pi \pi$ system may have final isospin 0 or 2 while the $K K$ can be in 0 and 1 states. Combining several measurements CLEO and

Table 10. CDF (preliminary) and FOCUS new measurements for $D^{0}$ decays.

\begin{tabular}{|c|c|c|}
\hline & $\frac{\Gamma\left(K^{+} K^{-}\right)}{\Gamma\left(K^{-} \pi^{+}\right)}(\%)$ & $\frac{\Gamma\left(\pi^{+} \pi^{-}\right)}{\Gamma\left(K^{-} \pi^{+}\right)}(\%)$ \\
\hline CDF & $9.38 \pm 0.18 \pm 0.10$ & $3.686 \pm 0.076 \pm 0.036$ \\
\hline FOCUS & $9.93 \pm 0.14 \pm 0.14$ & $3.53 \pm 0.12 \pm 0.06$ \\
\hline PDG & $10.84 \pm 0.26$ & $3.76 \pm 0.17$ \\
\hline
\end{tabular}


Table 11. For the isospin amplitude the experiments use new results from Table 10 combined with previous measurements on the channels. CLEO old results are listed for comparison. ${ }^{10}$

\begin{tabular}{|c|c|c|}
\hline$D \rightarrow \pi \pi$ & $\left|A_{2}\right| /\left|A_{0}\right|$ & $\left(\delta_{2}-\delta_{0}\right)\left(^{\circ}\right)$ \\
\hline FOCUS & $0.65 \pm 0.14$ & $83.6 \pm 10.0$ \\
\hline CLEO new & $0.421 \pm 0.040$ & $87.6 \pm 4.6$ \\
\hline CLEO old & $0.72 \pm 0.13 \pm 0.11$ & $82.0 \pm 7.5 \pm 5.2$ \\
\hline$D \rightarrow K K$ & $\left|A_{1}\right| /\left|A_{0}\right|$ & $\left(\delta_{1}-\delta_{0}\right)\left({ }^{\circ}\right)$ \\
\hline FOCUS & $0.56 \pm 0.04$ & $37.1 \pm 7.5$ \\
\hline CLEO old & $0.61_{0.10}^{+0.11}$ & $28.4_{9.7}^{12.1}$ \\
\hline
\end{tabular}

FOCUS estimate the ratios of amplitudes and phases differences listed on Table 11. The large phase differences denote the significance of FSI.

The full amplitude analysis of 4-body decay modes is very complex and not much information on the resonant substructure of such decays is available. If the dominance of quasi-two body decays, observed in the 3-body final state, is confirmed for the case of multi-body decays, then one can take a more complete comparison with theoretical models, developed mainly to describe the two-body and quasi-two-body decay modes.

FOCUS has reported on new signals for $D^{0} \rightarrow$ $K^{-} K^{+} K^{-} \pi^{+} ; K^{+} K^{-} \pi^{+} \pi^{-}$and $\pi^{+} \pi^{-} \pi^{+} \pi^{-28}$ For these they measure the branching ratio relative to the dominant $D^{0} \rightarrow K^{-} \pi^{+} \pi^{-} \pi^{+}$listed in Table 12 along with previous measurements listed in the PDG. The full coherent amplitude analysis for the channel $D^{0} \rightarrow K^{-} K^{+} K^{-} \pi^{+}$is now released. ${ }^{29}$ The formalism used is a straightforward extension of that described above for the 3-body decays, a coherent sum of Breit-Wigner resonances modulated by angular distribution functions and a constant non-resonant contribution. There are five degrees of freedom, and in principle, many intermediate states possibilities. This decay is Cabibbo-favored but phase space and $s \bar{s}$ creation suppressed. The analysis is based on a sample of 139 signal and 65 estimated background events. While a large number of intermediate states could lead to this final state, phase space restricts the possible contributions. The only resonant mode with nominal mass within the phase space is the $\phi K^{-} \pi^{+}$. Wide resonances without a particular angular distribution such as $\bar{K}^{0}(1430) K^{+} K^{-}$or $\kappa K^{+} K^{-}$are hard to distinguish from the NR contribution. On the other hand modes with $\bar{K}^{0}(892)$ with nominal mass just above the kinematic limit have a characteristic angular distribution as a signature and can be identified. The final solution for the best fit is
Table 12. $D^{0}$ branching ratios relative to $D^{0} \rightarrow K^{+} \pi^{+} \pi^{-} \pi^{+}$.

\begin{tabular}{|c|c|c|}
\hline mode & FOCUS & PDG \\
\hline$\left[K^{-} K^{+} \pi^{-} \pi^{+}\right]$ & $2.97 \pm 0.10$ & $3.34 \pm 0.28$ \\
\hline$\left[K^{-} K^{+} K^{-} \pi^{+}\right]$ & $0.257 \pm 0.034 \pm 0.023$ & $0.32 \pm 0.09$ \\
\hline$\left[\pi^{-} \pi^{+} \pi^{-} \pi^{+}\right]$ & $8.66 \pm 0.12$ & $9.8 \pm 0.6$ \\
\hline
\end{tabular}

Table 13. FOCUS best solution for the $D^{0} \rightarrow K^{-} K^{+} K^{-} \pi^{+}$ amplitude analysis.

\begin{tabular}{|c|c|c|}
\hline Component & Phase $\left(^{\circ}\right)$ & Fit fraction\% \\
\hline$\phi \bar{K}^{* 0}(892)$ & $0($ fixed $)$ & $48 \pm 6 \pm 1$ \\
\hline$\phi K^{-} \pi^{+}$ & $194 \pm 24 \pm 8$ & $18 \pm 6 \pm 4$ \\
\hline $\bar{K}^{* 0}(892) K^{-} K^{+}$ & $225 \pm 15 \pm 4$ & $20 \pm 7 \pm 2$ \\
\hline$N . R$. & $278 \pm 16 \pm 42$ & $15 \pm 6 \pm 2$ \\
\hline
\end{tabular}

listed in Table 13. Notice that the two vector mode $\bar{K}^{* 0}(892) \phi$ dominates the decay. Similar analysis for the remaining signals are expected soon.
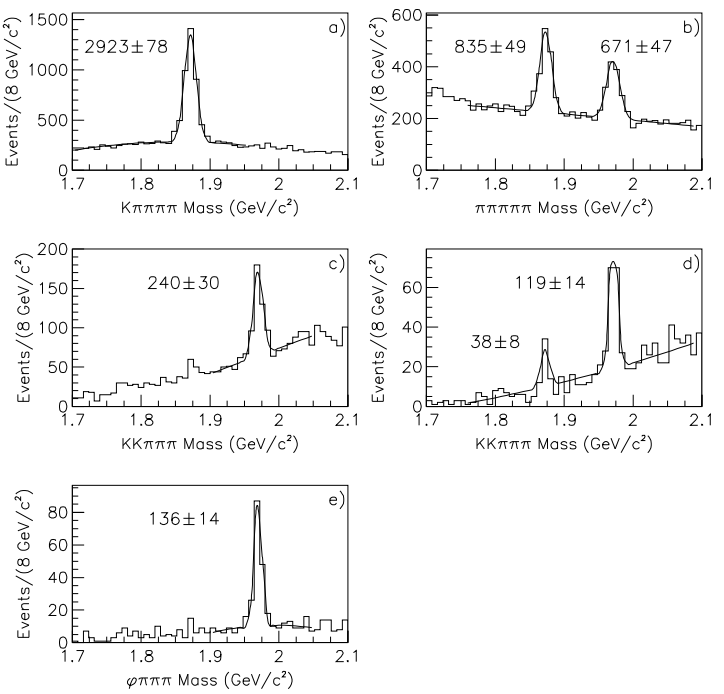

Figure 14. FOCUS five-body signals. Sub-resonant analyses were done for signals in a) and c). c) $K^{-} K^{+} \pi^{+} \pi^{+} \pi^{-}$optimization for $D_{s}^{+}$. d) Optimization for the first observation of the channel $D^{+} \rightarrow K^{-} K^{+} \pi^{+} \pi^{+} \pi^{-}$.

FOCUS have also recently published the 5body decays of $D^{+}$and $D_{s}^{+}$into kaons and pions. ${ }^{30}$ The inclusive branching ratios of five modes including the first evidence for the decay mode $D^{+} \rightarrow$ $K^{-} K^{+} \pi^{+} \pi^{+} \pi^{-}$are listed in Table 14 and the signals are in Fig. 14. They study the resonant substructure of the decays $D^{+} \rightarrow K^{-} \pi^{+} \pi^{+} \pi^{+} \pi^{-}$and $D_{s}^{+} \rightarrow K^{-} K^{+} \pi^{+} \pi^{+} \pi^{-}$. The full coherent amplitude analysis was not attempted, rather they used a simplified approach where the final state is assumed 
Table 14. FOCUS relative branching ratio for 5-body $D^{+}$and $D_{s}^{+}$decay modes.

\begin{tabular}{|c|c|}
\hline Mode & FOCUS \\
\hline$\frac{\Gamma\left(D^{+} \rightarrow K^{-} \pi^{+} \pi^{+} \pi^{+} \pi^{-}\right)}{\Gamma\left(D^{+} \rightarrow K^{-} \pi^{+} \pi^{+}\right)}$ & $0.058 \pm 0.002 \pm 0.006$ \\
\hline$\frac{\Gamma\left(D^{+} \rightarrow \pi^{+} \pi^{+} \pi^{+} \pi^{-} \pi^{-}\right)}{\Gamma\left(D^{+} \rightarrow K^{-} \pi^{+} \pi^{+} \pi^{+} \pi^{-}\right)}$ & $0.290 \pm 0.017 \pm 0.011$ \\
\hline$\frac{\Gamma\left(D_{s}^{+} \rightarrow \pi^{+} \pi^{+} \pi^{+} \pi^{-} \pi^{-}\right)}{\Gamma\left(D_{s}^{+} \rightarrow K^{-} K+\pi^{+}\right)}$ & $0.145 \pm 0.011 \pm 0.010$ \\
\hline$\frac{\Gamma\left(D_{s}^{+} \rightarrow K^{+} K^{-} \pi^{+} \pi^{+} \pi^{-}\right)}{\Gamma\left(D_{s}^{+} \rightarrow K^{-} K^{+} \pi^{+}\right)}$ & $0.150 \pm 0.019 \pm 0.025$ \\
\hline$\frac{\Gamma\left(D_{s}^{+} \rightarrow \phi \pi^{+} \pi^{+} \pi^{-}\right)}{\Gamma\left(D_{s}^{+} \rightarrow \phi \pi^{+}\right)}$ & $0.249 \pm 0.024 \pm 0.021$ \\
\hline$\frac{\Gamma\left(D^{+} \rightarrow K^{+} K^{-} \pi^{+} \pi^{+} \pi^{-}\right)}{\Gamma\left(D^{+} \rightarrow K^{-} \pi^{+} \pi^{+} \pi^{+} \pi^{-}\right)}$ & $0.040 \pm 0.009 \pm 0.019$ \\
\hline
\end{tabular}

to be an incoherent superposition of sub-resonant decays involving vector resonances. In both cases the non-resonant component is small.

\section{Conclusions}

The small fraction of recent results discussed here is illustrative of the picture drawn in the introduction: there is a lot to be understood and the conditions are favorable for the development of the field. Several experiments, not specially designed to do charm physics, have now some impressive quality charm data.

\section{References}

1. S. Bianco, F. L. Fabbri, D. Benson and I. Bigi, hep-ex/0309021.

2. FOCUS Collab., Phys. Lett. B 535, 43-51 (2002), hep-ex/0203031.

3. Babar Collab., Phys. Rev. Lett. 90, 242001 (2003), hep-ex/0304021 and hep-ex/0309028.

4. CLEO Collab., hep-ex/0305100.

5. Belle Collab., hep-ex/0307052, hep-ex/0307041.

6. SELEX Collab., Phys. Rev. Lett. 89, 112001 (2003).

7. J. S. Russ for SELEX Collab., at the Fermilab Wine\&Cheese seminar $(6 / 13 / 03)$.
8. Belle Collab., hep-ex/0307021.

9. Delphi Collab., Phys. Lett. B 426, (1998).

10. Particle Data Group, Phys. Rev. D 66, 010001 (2002).

11. R.Kutschke for FOCUS Collab., Frontiers in Contemporary Physics II, Nashville March (2001).

12. Barnes, Close and Lipkin, hep-ph/0305025; Bardeen, Eichten and Hill, hep-ph/0305049; Nowak, Rho and Zahed, hep-ph/0307102; Colangelo and De Fazio, hep-ph/0305140; Van Beveran and Rupp, hep-ph/0308166; Dai, Huang and Zhu, hep-ph/0306274; Browder, Pakvasa and Petrov, hep-ph/0307054.

13. A. Palano for BaBar Collab., talk at Physics in Collision, Germany (2003), hep-ex/0309028.

14. P. Sheldon for FOCUS Collab., talk at International Workshp on Frontier Science 2002, Frascati, Italy.

15. E791 Collab., Phys. Rev. Lett. 86, 770 (2001).

16. Bediaga and Miranda, Phys. Lett. B 550, 135 (2002), hep-ph/0211078.

17. Bediaga for E791 Collab., talk at Scalar Meson Workshop, Utica, May (2003), hep-ex/0307008.

18. CLEO Collab., Phys. Rev. Lett. 89, 251802 (2002).

19. V.V. Frolov for CLEO Collab., hep-ex/0306048.

20. B. Aubert for Babar Collab., ICHEP2002 hep-ex/0207089.

21. E791 Collab., Phys. Rev. Lett. 89, 121801 (2002).

22. E691 Collab., Phys. Rev. D 48, 56 (1995); E687 Collab., Phys. Lett. B 331, 217 (1994).

23. Gobel for E791 Collab., talk at Scalar Meson Workshop, Utica, May (2003), hep-ex/0307003.

24. LASS Collab., Nucl. Phys. B 296, 493 (1988).

25. FOCUS Collab., Phys. Lett. B 555, 167 (2003), hep-ex/0212058.

26. CLEO Collab., CLEO CONF 03-02, EPS-371.

27. I. Furic for CDF Collab., XVII Rencontres de Physic de la Valle d'Aoeste, March 2003, Italy.

28. K. Stenson, talk "Recent Results in Charm Decays", APS (2003).

29. FOCUS Collab., hep-ex/0308054.

30. FOCUS Collab., Phys. Lett. B 561, 225 (2003), hep-ex/02011056. 


\section{DISCUSSION}

Questioner: I want to comment on your $\sigma(500)$. What you have done is really what is traditionally done in partial wave analyses. One does the analysis in partial waves and after obtaining the result, one fits it with a Breit-Wigner or a modified Breit-Wigner. So it is a very conventional sort of thing.

Jussara de Miranda: Not in a Dalitz plot.

Questioner: You can take the events out of a Dalitz plot and do it if you like.

Jussara de Miranda: We have tried this, it is much harder, you have to have one parameter for each slice. I've never seen this in a Dalitz plot. There are people in our collaboration trying to take this out. I understand that is what is done in scattering experiments and traditional partial wave analyses.

Questioner: In a Dalitz plot you cannot take the whole thing as there is crossing and this and that, but really this is very equivalent to what you have done.

Jussara de Miranda: Yes, the idea is the same, the way to go is the difference. 